\title{
Network pharmacological systems study of Huang-Lian-Tang in the treatment of glioblastoma multiforme
}

\author{
WANGRUI LIU ${ }^{1,2^{*}}$, WENHAO XU ${ }^{3,4^{*}}$, CHUANYU LI ${ }^{1,2^{*}}$, JUNFEI XU $^{5}$, KE HUANG $^{6}$, \\ RENYUE HU ${ }^{7}$, HAINENG HUANG ${ }^{1,2}$ and XIAOJUAN LIU ${ }^{8}$
}

\begin{abstract}
${ }^{1}$ Department of Neurosurgery, Affiliated Hospital of Youjiang Medical University for Nationalities; ${ }^{2}$ Clinical College of Youjiang Medical University for Nationalities, Baise, Guangxi 533000; ${ }^{3}$ Department of Urology, Fudan University Shanghai Cancer Center; ${ }^{4}$ Department of Oncology, Shanghai Medical College, Fudan University, Shanghai 20032; ${ }^{5}$ Department of General Surgery, Affiliated Hospital of Nantong University, Nantong, Jiangsu 226001; ${ }^{6}$ Department of Obstetrics and Gynecology, The Affiliated Obstetrics and Gynecology Hospital of Nanjing Medical University, Nanjing, Jiangsu 210004; ${ }^{7}$ Department of Endocrinology and Metabolism, Affiliated Hospital of Nantong University;

${ }^{8}$ Department of Pathogenic Biology, Medical College, Nantong University, Nantong, Jiangsu 226001, P.R. China
\end{abstract}

Received March 12,2020; Accepted August 18, 2020

DOI: $10.3892 / \mathrm{ol} .2020 .12279$

\begin{abstract}
Glioblastoma multiforme (GBM) has a poor prognosis and its recurrence and mortality rates are high. At present, there is no effective clinical method to control its progression and recurrence. Traditional Chinese Medicine has a high status not only in China, but also in the world. Certain drugs are also used in the clinical treatment of tumor diseases. In clinical practice, Huang-Lian-Tang (HLT) has proven efficacy in treating brain diseases and preventing tumor recurrence. However, the mechanisms of action have remained elusive. The present study explored the potential mechanisms of HLT in the treatment of gliomas based on network pharmacology. First, information on the composition of HLT was obtained from the Traditional Chinese Medicine Systems Pharmacology Database and Analysis Platform, and the composition and targets of the chemical substances contained in the herbs were analyzed. Subsequently, a pharmacological interaction network for HLT was built. Furthermore, the expressed genes
\end{abstract}

Correspondence to: Professor Haineng Huang, Department of Neurosurgery, Affiliated Hospital of Youjiang Medical University for Nationalities, 18 Zhongshan 2nd Road, Baise, Guangxi 533000, P.R. China

E-mail: bshuanghn@163.com

Professor Xiaojuan Liu, Department of Pathogenic Biology, Medical College, Nantong University, 19 Qixiu Road, Nantong, Jiangsu 226001, P.R. China

E-mail:1xj@ntu.edu.cn

*Contributed equally

Key words: Huang-Lian-Tang, network pharmacological system, glioblastoma multiforme, bioinformatics, treatment, Traditional Chinese Medicine of patients with GBM were obtained from Gene Expression Omnibus database and screened. A protein-protein interaction network was then constructed for both sets of data and they were combined with a topology method for analysis. Finally, the screened genes were subjected to enrichment analysis and pathway analysis. A total of 386 candidate targets and 7 Kyoto Encyclopedia of Genes and Genomes (KEGG) pathways were screened, which were mainly associated with amino acid metabolism. Gene Ontology enrichment analysis and KEGG signal pathway analysis indicated that these targets are involved in anti-apoptosis, anti-oxidative stress, multicellular biological processes and other physiological and pathological processes related to the occurrence and development of GBM. In conclusion, the present results indicated that the mechanisms of action of HLT against GBM involve multiple targets and signaling pathways that are related to tumorigenesis and progression. The present study not only provided a novel theoretical basis for Traditional Chinese Medicine to treat tumors but also novel ideas for the treatment of GBM.

\section{Introduction}

Gliomas are the most common central nervous system tumors in adults, accounting for $44.69 \%$ of intracranial tumors and $70 \%$ of them are malignant gliomas (1). The incidence of glioma is $\sim 6.04$ cases per 100,000 individuals in 2013, and although this incidence is not high, the mortality rate is very high (2), and therefore, glioma may be considered dangerous. Due to the particularity of the intracranial structure, gliomas exhibit high invasiveness, a high recurrence rate and high resistance to traditional radiochemotherapy, and under the most ideal treatment conditions, the prognosis of patients with glioma is still not ideal (3). The survival time of patients with GBM is only $12-15$ months, the vast majority of patients die within 2 years and the 5 -year survival rate is $<5 \%(2,4)$. Due to the characteristics of invasive growth and progressive malignancy of gliomas, it is difficult to completely remove 
the tumor with surgical treatment. Adjuvant therapies, such as radiation therapy and chemotherapy can only eliminate tumor cells or delay recurrence to a certain extent and can also cause certain damage to the patient's body $(5,6)$. Although numerous advances have been made in the standardized as well as individualized comprehensive treatment of malignant gliomas, the clinical efficacy remains limited (7).

In the clinic, certain Traditional Chinese Medicine prescriptions have been used to treat serious diseases such as tumors (8). Huang-Lian-Tang (HLT) is a classic Chinese herbal formula. The major ingredients are Coptis chinensis, Hebeninos commutaverunt and Gingiberi exaruit. In China and other Asian countries, HLT has a certain or a subtle effect in numerous diseases (such as tumor, diabetes, arthritis, ischemic stroke and liver disease) (9). Previous studies have indicated that activation of immune cells and metabolic reprogramming the inflammatory response are involved in the mechanism of action (9-11). Studies have also suggested that one of the major components of HLT, Huanglian, has a valuable effect in the treatment of Alzheimer's disease and brain injury $(12,13)$. Furthermore, Huanglian has also been effective in treating certain types of cancer (14-17).

To improve the treatment of gliomas, the present study turned to Chinese medicine to identify whether it is able to inhibit key molecular targets and signaling pathways associated with the occurrence aggressive growth behavior of gliomas to provide novel approaches for their clinical treatment. The anti-proliferative effect of HLT on cancer cell growth has been demonstrated in human myeloma cells (8-10). However, it has remained elusive whether HLT has any effect on gliomas and their therapeutic targets. In the present study, network pharmacological systems analysis technology was used to explore and analyze the multi-component, multi-target and multi-pathway interaction laws and regulatory networks involved in the inhibition of glioma cell growth, cell-cycle arrest and apoptosis induced by HLT. The present study lays a foundation for future in-depth study of the mechanism of action of HLT in the treatment of gliomas and the development of novel valuable drugs and may provide novel approaches for the clinical treatment of GBM.

\section{Materials and methods}

Data preparation. Drug data were obtained from the Traditional Chinese Medicines Systems Pharmacology database (TCMSP; http://tcmspw.com/tcmsp.php) (18). TCMSP is a unique systems pharmacology platform of Chinese herbal medicines that captures the relationships between drugs, targets and diseases. The database includes chemicals, targets and drug-target networks, and associated drug-target-disease networks, as well as pharmacokinetic properties of natural compounds, including oral bioavailability, drug-likeness, intestinal epithelial and blood-brain-barrier permeability, and aqueous solubility.

Enrichment analysis was performed using the Database for the Annotation, Visualization and Integrated Discovery database (DAVID; https://david.ncifcrf.gov/) using tumor gene data from the National Center for Biotechnology Information (NCBI) Gene Expression Omnibus (GEO) database (https://www.ncbi.nlm.nih.gov/geo).
Traditional Chinese Medicine ingredient screening and target detection. In order to obtain the molecular drug composition of HLT, the TCMIP database was searched to retrieve the name of each active component included in HLT and their chemical structural similarity to commercially available drugs was compared using the US Food and Drug Administration (https://www.fda.gov/). The standard of oral bioavailability $\geq 30 \%$ and drug-likeness $\geq 0.18$ was used to screen Chinese medicine ingredients and target analysis was performed. Using the aforementioned criteria, pharmaceutical ingredients that serve a therapeutic role were screened.

Gene screening. The GEO datasets (GSE108474, GSE109857, GSE143263, GSE145940 and GSE150956) included for glioblastoma cells or tissues were compared with those for normal brain tissue in order to identify differentially expressed genes in glioma. In addition, only datasets containing more than 20 samples were selected. The titles and summaries of 350 potentially relevant datasets were screened to identify eligible datasets for further evaluation. Finally, only GSE108474 (72 samples) were selected for further analysis. The GSE108474 dataset based on the GPL570 platform was downloaded from the NCBI GEO database, containing the data of 20 normal brain tissues and 52 tumor samples. The Limma version 3.11 software package (https://bioconductor.org/packages/limma/) was used to screen genes that were differentially expressed between the high-risk group and the low-risk group (adjusted P-value $<0.01$, fold change at least $\mathrm{x} 2$ ).

Drug-associated active ingredient inspection and target screenings. The names of the major ingredients of HLT, namely Coptis chinensis, ume and dried ginger, were inputted in the TCMSP database and the chemical composition of each drug was retrieved. The screening conditions were based on oral bioavailability $(\mathrm{OB} ; \geq 30 \%)$ and drug-likeness ( $\mathrm{DL} \geq 0.18$ ), so as to obtain the drug composition and targets. Numerous recent studies have shown that the screening criteria can screen out effective pharmaceutical ingredients (19-22).

Drug target-GBM network construction. The drug- disease network was constructed using the Bisogenet (23) plug-in of Cytoscape software (version 3.7.2) for drug targets that were active through TCMSP and the differentially expressed genes in glioma identified from the GEO dataset. It contains six PPI databases, including their Interaction Protein Database, the Biological Universal Repository of Interaction Data Sets, Human Protein Reference Database, the IntAct Molecular Interaction Database, the Biomolecular Interaction Network Database and the Molecular Interaction Action database. Two PPI interactive networks were built and illustrated using Cytoscape software, including the screened HLT target and the known GBM target. After combining these two networks into candidate networks, topology analysis was used to gradually screen out the central network.

Network topology analysis. The analysis was performed by using the CytoNCA (24) plug-in in Cytoscape software by determining these 6 metrics: Degree centrality (DC), topology intermediateness (BC), closeness centrality (CC), feature vector centrality (EC), local average connectivity (LAC), and network 


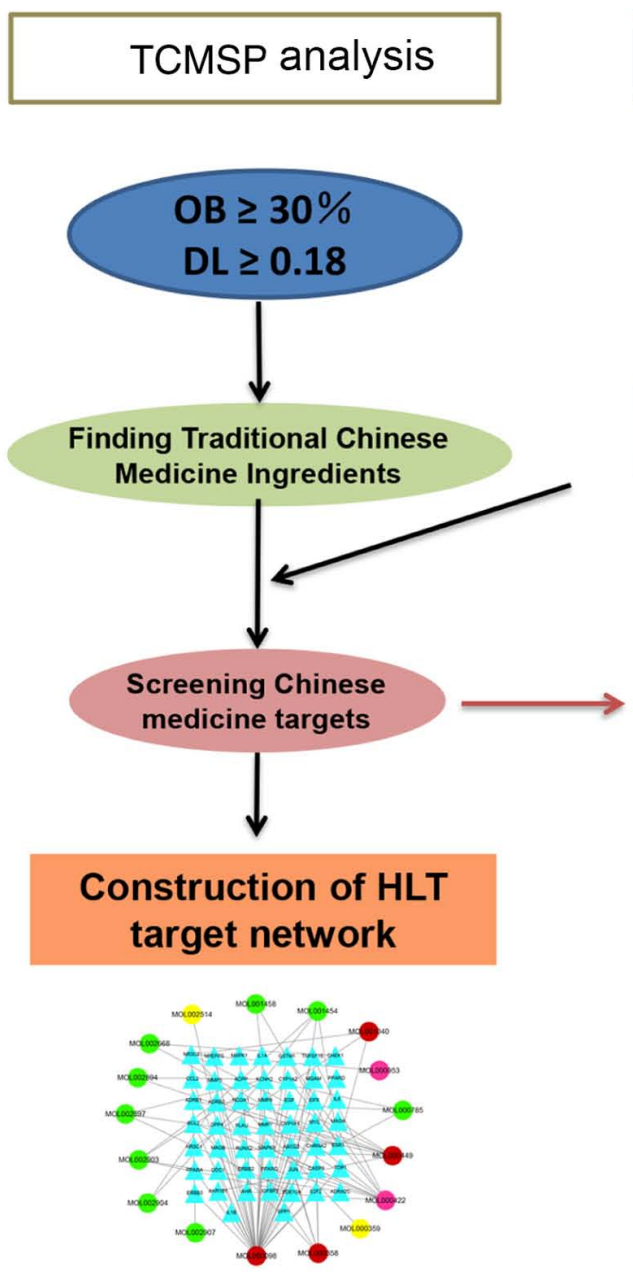

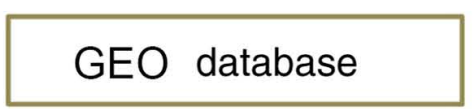
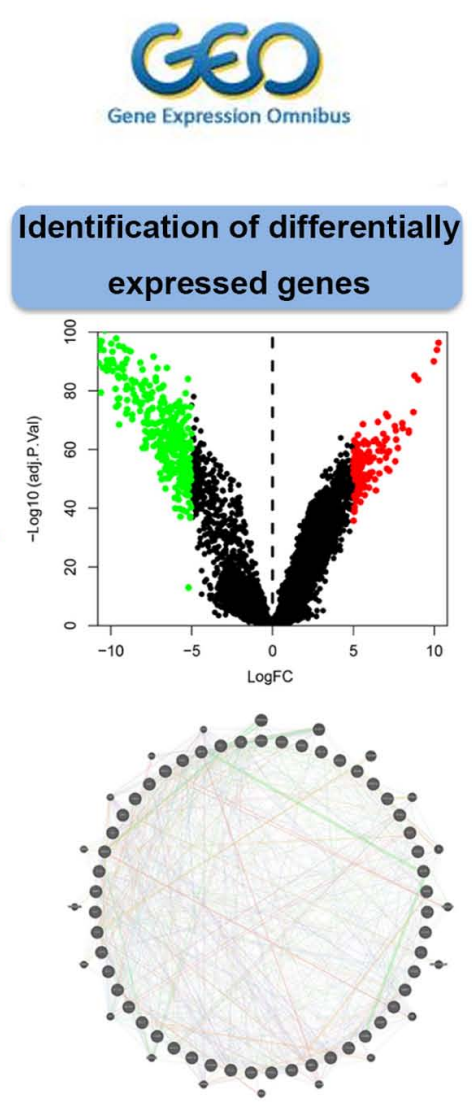

Networks

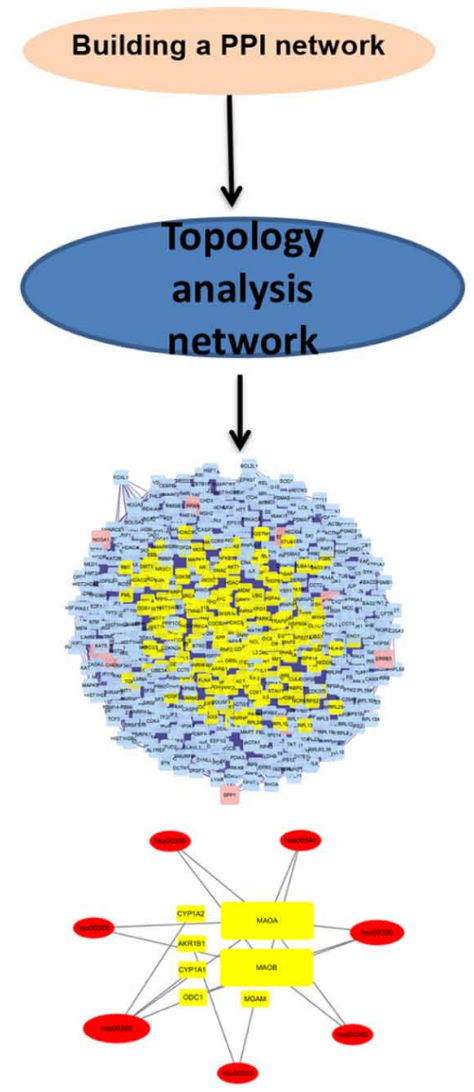

Figure 1. Flowchart of the systematic profiling of the alternative splicing in glioblastoma multiforme in the present study. TCMSP, Traditional Chinese Medicine Systems Pharmacology Database and Analysis Platform; OB, oral bioavailability; DL, drug-likeness; GEO, Gene Expression Omnibus database; PPI, protein-protein interaction; HLT, Huang-Lian-Tang.

centrality (NC). The six metrics provide an in-depth analysis of the attributes of all nodes in the interactive network. The definition and calculation equations of all parameters reflect the significance of the nodes. A higher quantitative value indicates a greater significance in the network. Through the above methods, the desired central network was gradually selected.

Screening the collection of core Protein-Protein Interaction (PPI) networks. The tightly connected area of molecular complexes in the huge PPI network obtained was defined as topological modules with pure network characteristics $(25,26)$. The aggregation of similar nodes and related nodes of drugs in the same group are defined as the pharmacology modules. Networks that destroy cell functions or lead to the GBM phenotype are defined as pathogenic modules. In the topology analysis, pharmacology modules and pathogenic modules are defined in the same network. Thus, the pathogenic module is considered to play a role in interference and destruction. The final core PPI network cluster was obtained by analyzing the corresponding network using the MCODE plug-in in Cytoscape software (26).

Gene ontology $(G O)$ and pathway enrichment analysis. Functional enrichment analyses of the screened genes were performed through GO analysis (27) in three categories, namely biological processes (BP), cellular component (CC) and molecular function (MF), and DAVID was used to perform gene and genomic encyclopedia of the Kyoto Encyclopedia of Genes and Genomes (KEGG) signaling pathway analysis. Functional terms and pathways with $\mathrm{P} \leq 0.05$ were considered important. The ggplot 2 plugin package for $\mathrm{R}$ software (vision 3.6.2) was used to visualize the results.

\section{Results}

Research process. The present study was divided into three phases in sequence, and Fig. 1 presents all of the processes in the systematic analysis. First, the drug components of HLT were downloaded from TCMIP and the components were analyzed to build a drug network. Furthermore, the data of patients with GBM were downloaded from GEO and the differentially expressed genes were screened to construct a network. Next, the two networks were merged together and a topology analysis was applied to filter out the desired core network. The network contained a total of 171 nodes and 6,309 edges. Finally, GO and KEGG analyses were performed on these targets to determine the mechanism of action of HLT against GBM. 
Table I. Top 10 most upregulated genes.

\begin{tabular}{lcccccc}
\hline Gene name & logFC & Average expression & $\mathrm{t}$ & P-value & Adjusted P-value & B \\
\hline $\begin{array}{l}\text { RPS25 (Ribosomal } \\
\text { Protein S25) }\end{array}$ & 10.26617 & 10.03903 & 186.0101 & $5.71 \times 10^{-100}$ & $3.86 \times 10^{-97}$ & 200.7867 \\
$\begin{array}{l}\text { STARD7 (StAR Related } \\
\text { Lipid Transfer Domain }\end{array}$ & 10.16257 & 9.964207 & 171.7686 & $1.96 \times 10^{-97}$ & $9.94 \times 10^{-95}$ & 197.2763 \\
$\begin{array}{l}\text { Containing 7) } \\
\text { YTHDF1 (YTH }\end{array}$ & 9.965356 & 9.821776 & 151.0904 & $2.36 \times 10^{-93}$ & $8.60 \times 10^{-91}$ & 191.1616 \\
$\begin{array}{l}\text { N6-Methyladenosine } \\
\text { RNA Binding Protein 1) }\end{array}$ & & & & & & \\
QRICH1 (Glutamine & 8.783873 & 8.968483 & 128.8207 & $2.76 \times 10^{-88}$ & $6.65 \times 10^{-86}$ & 182.8256 \\
Rich 1) & & & & & \\
CRKL (CRK Like & 9.000407 & 9.124869 & 123.2493 & $7.00 \times 10^{-87}$ & $1.51 \times 10^{-84}$ & 180.383 \\
$\begin{array}{l}\text { Proto-Oncogene, } \\
\text { Adaptor Protein) }\end{array}$ & & & & & \\
$\begin{array}{l}\text { KHDC4 (KH Domain } \\
\text { Containing 4, Pre-MRNA }\end{array}$ & 8.712287 & 8.916781 & 86.2513 & $1.42 \times 10^{-75}$ & $1.58 \times 10^{-73}$ & 158.9653 \\
$\begin{array}{l}\text { Splicing Facto) } \\
\text { MRPL33 (Mitochondrial }\end{array}$ & 7.022553 & 7.696419 & 84.3207 & $7.39 \times 10^{-75}$ & $7.83 \times 10^{-73}$ & 157.5233 \\
$\begin{array}{l}\text { Ribosomal Protein L33) } \\
\text { SYF2 (SYF2 Pre-MRNA }\end{array}$ & 7.127974 & 7.772555 & 82.1461 & $4.94 \times 10^{-74}$ & $5.05 \times 10^{-72}$ & 155.8492 \\
$\begin{array}{l}\text { Splicing Factor) } \\
\text { PYGO2 (Pygopus Family }\end{array}$ & 6.51068 & 7.326732 & 77.2753 & $4.18 \times 10^{-72}$ & $3.91 \times 10^{-70}$ & 151.8939 \\
$\begin{array}{l}\text { PHD Finger 2) } \\
\text { PHF2 (PHD Finger }\end{array}$ & 8.01312 & 8.411828 & 76.1175 & $1.25 \times 10^{-71}$ & $1.12 \times 10^{-69}$ & 150.9093 \\
Protein 2) & & & & & & \\
\hline
\end{tabular}

There were a total of 2,751 upregulated genes. FC, fold change.

Identification of differentially expressed genes in GBM. The dataset GSE108474 was selected and downloaded from the GEO database as a sample, which contained the data of 20 normal tissues and 52 tumor tissues. In order to screen for differentially expressed genes, a differential expression analysis on the data was performed obtained using the limma software package. By performing this analysis, it was determined that compared with normal brain tissue samples, a total of 5,491 genes were significantly differentially expressed in GBM, 2,751 of which were upregulated genes and 2,740 were downregulated genes. Tables I and II list the top 10 most upregulated and downregulated genes, respectively. According to the fold change, Ribosomal Protein S25 (RPS25), StAR Related Lipid Transfer Domain Containing 7 (STARD7) and YTH N6-Methyladenosine RNA Binding Protein 1(YTHDF1) are the three genes with the highest degree of upregulation, while High Mobility Group Nucleosomal Binding Domain 2 (HMGN2), Staufen Double-Stranded RNA Binding Protein 1 (STAU1) and Ferritin Heavy Chain 1 Pseudogene 5 (FTH1P5) were the three genes with the highest degree of downregulation. A heat map (Fig. 2A) and a volcano plot (Fig. 2B) were drawn to visualize the differentially expressed genes.

Chemical composition of HLT and traditional Chinese medicine target prediction. A total of 468 chemical ingredients of
7 Chinese herbs in HLT were collected through the TCMIP database of Chinese medicinal ingredients, and similarity scores with drugs in the DrugBank database were obtained. Similar drugs with a score $\geq 0.8$ were considered to be highly similar to the chemical ingredients contained in HLT. A total of 3,939 'component-target' pairs of HLT were retrieved. Among them, there were 14 components of Coptis chinensis with 87 predicted targets, 38 components of dried ginger with 26 predicted targets, 12 components of cinnamon sticks with 57 predicted targets, 41 components of Pinellia ternata with 237 predicted targets, 72 components of jujube with 1,172 predicted targets, 158 components of ginseng with 2,000 predicted targets and 133 components of licorice with 360 predicted targets. The components and targets in the obtained associations were assigned to the single drugs contained in HLT to obtain the target information corresponding to each drug. Among them, Coptis chinensis had 67 component targets and Guizhi had 46 component targets. Dried ginger, Pinellia ternata, ginseng, jujube and licorice each contained 7, 196, 876, 429, 115 component targets. The common targets between the two constituent drugs were analyzed (Table III). The results suggested that there were different numbers of common targets among the 7 components, of which 57 were common targets of Coptis chinensis and Ginseng, accounting for $85.1 \%$ of the total number of targets of Coptis chinensis. There were 152 targets for Pinellia ternata and ginseng. 
Table II. Top 10 most downregulated genes.

\begin{tabular}{|c|c|c|c|c|c|c|}
\hline Id & $\log \mathrm{FC}$ & Average expression & $\mathrm{t}$ & P-value & Adjusted P-value & $\mathrm{B}$ \\
\hline $\begin{array}{l}\text { HMGN2 (High Mobility } \\
\text { Group Nucleosomal } \\
\text { Binding Domain 2) }\end{array}$ & -12.813 & 3.559164 & -296.316 & $8.37 \times 10^{-115}$ & $1.19 \times 10^{-110}$ & 216.6827 \\
\hline $\begin{array}{l}\text { STAU1 (Staufen } \\
\text { Double-Stranded RNA } \\
\text { Binding Protein 1) }\end{array}$ & -11.6436 & 3.234342 & -290.265 & $3.80 \times 10^{-114}$ & $2.70 \times 10^{-110}$ & 216.1427 \\
\hline $\begin{array}{l}\text { FTH1P5 (Ferritin Heavy } \\
\text { Chain } 1 \text { Pseudogene 5) }\end{array}$ & -12.9861 & 3.607250 & -272.269 & $4.17 \times 10^{-112}$ & $1.98 \times 10^{-108}$ & 214.3746 \\
\hline $\begin{array}{l}\text { CHERP (Calcium } \\
\text { Homeostasis Endoplasmic } \\
\text { Reticulum Protein) }\end{array}$ & -11.5667 & 3.212962 & -260.047 & $1.21 \times 10^{-110}$ & $3.86 \times 10^{-107}$ & 213.0173 \\
\hline $\begin{array}{l}\text { RP5-930J4.4 (RP5 } \\
\text { Pre-MRNA Splicing Factor) }\end{array}$ & -12.3119 & 3.419980 & -259.647 & $1.36 \times 10^{-110}$ & $3.86 \times 10^{-107}$ & 212.9705 \\
\hline $\begin{array}{l}\text { RPL34 (Ribosomal } \\
\text { Protein L34) }\end{array}$ & -12.4815 & 3.467075 & -251.274 & $1.51 \times 10^{-109}$ & $3.56 \times 10^{-106}$ & 211.9538 \\
\hline $\begin{array}{l}\text { UBA3 (Ubiquitin Like } \\
\text { Modifier Activating } \\
\text { Enzyme 3) }\end{array}$ & -11.0044 & 3.056778 & -243.291 & $1.61 \times 10^{-108}$ & $2.96 \times 10^{-105}$ & 210.9144 \\
\hline $\begin{array}{l}\text { RPL27A (Ribosomal } \\
\text { Protein L27a) }\end{array}$ & -12.5865 & 3.496262 & -243.174 & $1.67 \times 10^{-108}$ & $2.96 \times 10^{-105}$ & 210.8986 \\
\hline $\begin{array}{l}\text { BTBD1 (BTB Domain } \\
\text { Containing 1) }\end{array}$ & -11.792 & 3.275543 & -241.309 & $2.93 \times 10^{-108}$ & $4.63 \times 10^{-105}$ & 210.645 \\
\hline $\begin{array}{l}\text { NSUN2 (NOP2/Sun } \\
\text { RNA Methyltransferase 2) }\end{array}$ & -10.8641 & 3.017812 & -240.118 & $4.21 \times 10^{-108}$ & $5.99 \times 10^{-105}$ & 210.481 \\
\hline
\end{tabular}

There were a total of 2,740 downregulated genes. FC, fold change.

Compound regulation network of HLT. A compound regulation network of the active components and molecular relationships of HLT was built and 766 related events and 68 nodes were obtained. There are 17 drug nodes (round nodes; dried ginger is yellow, Coptis is green, ume is pink, and various drug combinations are red) and 51 molecular nodes (triangular nodes). The core target network was mapped through Cytoscape (Fig. 3).

Construction and analysis of PPI network. With the rise of network pharmacology, its role in systems biology research is increasing. In the present study, the HLT drug target network and the PPI network for GBM were fused (Fig. 4A and B). In order to reveal the pharmacological mechanism of HLT against GBM, Cytoscape software was used to combine two huge networks to build a new and complex network (2,954 nodes and 72,642 edges), which resembled a merge of the HLT and GBM networks (Fig. 4B). Subsequently, the CytoNCA plugin in Cytoscape was used to confirm the core goals. The selection criteria were set as follows: $\mathrm{BC}$ value $>450, \mathrm{CC}>0.6$, $\mathrm{DC}>70, \mathrm{EC}>0.02, \mathrm{NC}>20$ and $\mathrm{LAC}>18$ (Fig. 4C and D). Finally, a core network comprising 171 nodes and 6,309 edges was screened out.

Enrichment analysis and KEGG signaling pathways of candidate targets for HLT against glioma. The 386 candidate targets for HLT in treating GBM were subjected to a GO analysis in the DAVID database to explore the links between the functional units, their potential significance in the biological systems network. The mechanism of action is clarified through this functional enrichment analysis The GO terms were determined in the following categories: Biological processes (Fig. 5A), cellular component (Fig. 5B) and molecular function (Fig. 5C). In the category biological process, the candidate genes were associated with response to nutrient levels, response to steroid hormone, response to lipopolysaccharide and response to molecule of organism process. In the category cellular component, enriched terms were transcription factor complex, RNA polymerase II transcription factor complex, nuclear transcription factor complex, myelin sheath, nuclear chromatin and apical plasma membrane. Finally, in the category molecular function, genes were associated with repression of transcription factor binding and RNA polymerase II transcription factor binding. A KEGG enrichment analysis was also performed on these genes, revealing that they are mainly related to amino acid metabolism (Fig. 5D).

$K E G G$ relationship network construction. To clarify the overall mechanisms of HLT in the treatment of gliomas, the above-mentioned steps provided a complete approach based on the current knowledge of the pathogenesis of gliomas (Fig. 6). Through layer-by-layer screening, 7 genes (oval) and 7 KEGG 

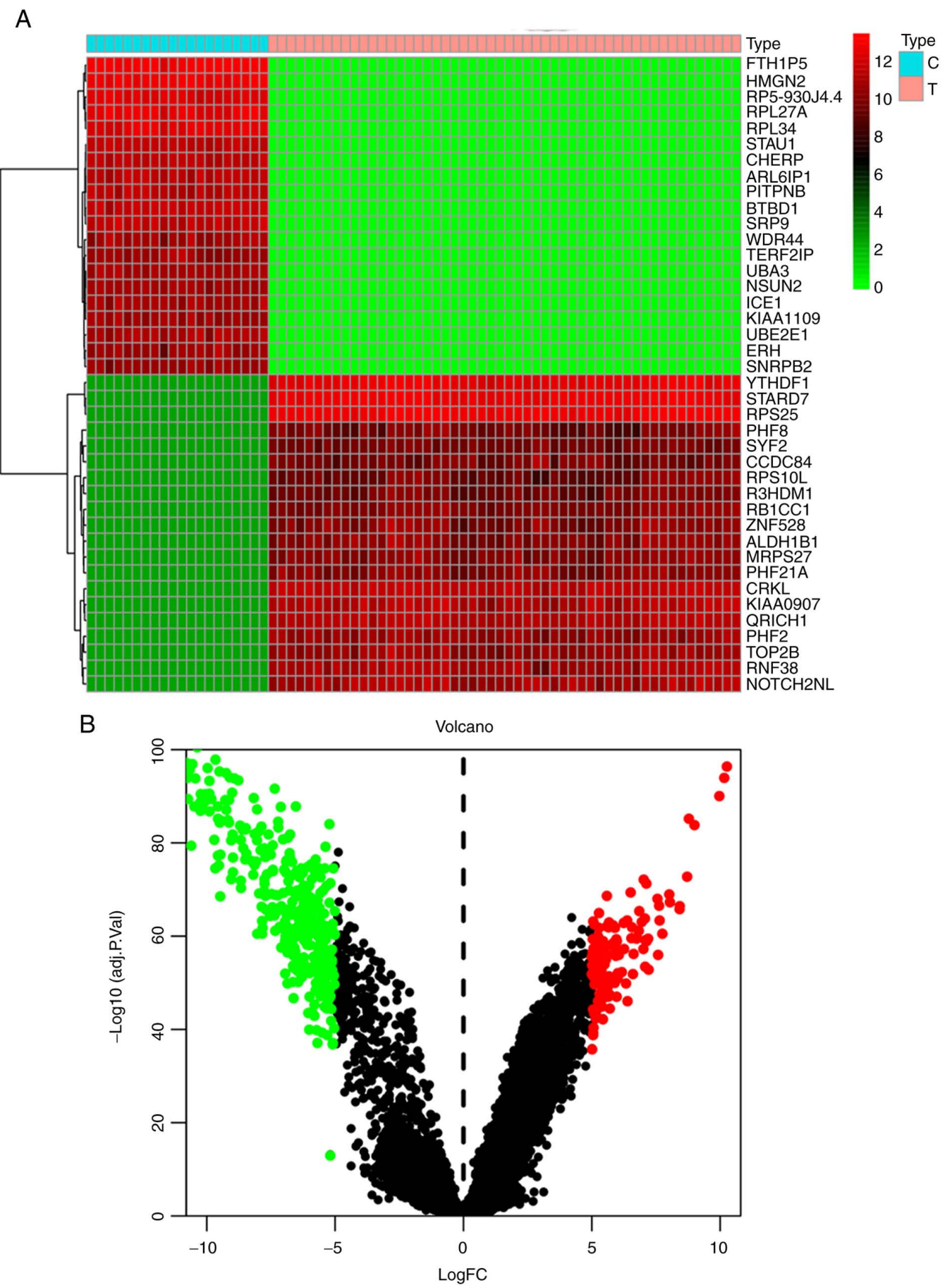

Figure 2. Heatmap and volcano plot of the differentially expressed genes. (A) Heatmap of the genes with differential expression between tumor and control samples. (B) Volcano plot. Black dots indicate genes that are not differentially expressed between 20 normal brain tissue samples and 52 tumor samples, and red and green dots indicate genes that are up- and down-regulated in tumor samples, respectively. FC, fold change; C, control; T, tumor; P.val, P-value.

signaling pathways (rectangular) associated with them were obtained; the size of the symbols is associated with the number of adjacent nodes. The 7 genes were MAOA (Monoamine Oxidase A), MAOB (Monoamine Oxidase B), MGAM (Maltase-Glucoamylase),CYP1A2 (Cytochrome P450 Family 1 Subfamily A Member 2), AKR1B1(Aldo-Keto Reductase Family 1 Member B), CYP1A1(Cytochrome P450 Family 1 Subfamily A Member 1) and ODC1(Ornithine Decarboxylase 1). The $7 \mathrm{KEGG}$ signaling pathways were hsa00380, hsa0052, hsa00260, hsa00330, hsa00340, hsa00350 and hsa00360.

\section{Discussion}

In the present study, target genes associated with GBM were first screened. It was analyzed which genes influence the occurrence, development and prognosis of GBM. The active components of HLT were also identified through an online pharmacology database. Combining the interaction networks of the two provided the potential mechanisms of action of HLT in the treatment of GBM. The present study provided a foundation for further research on Traditional Chinese Medicine treatments for GBM to improve the prognosis of affected 
Table III. Common pharmacology-targets between any two components of Huang-Lian-Tang.

\begin{tabular}{|c|c|c|c|c|c|c|c|}
\hline $\begin{array}{l}\text { Component } \\
\text { (target number) }\end{array}$ & $\begin{array}{c}\text { Dried } \\
\text { ginger (7) }\end{array}$ & $\begin{array}{l}\text { Ginseng } \\
\text { (876) }\end{array}$ & $\begin{array}{l}\text { Guizhi } \\
(46)\end{array}$ & $\begin{array}{l}\text { Jujube } \\
(429)\end{array}$ & $\begin{array}{c}\text { Coptis } \\
\text { chinensis (67) }\end{array}$ & $\begin{array}{c}\text { Pinellia } \\
\text { (196) }\end{array}$ & $\begin{array}{l}\text { Licorice } \\
\text { (115) }\end{array}$ \\
\hline Dried ginger (7) & l & 7 & 0 & 1 & 0 & 1 & 0 \\
\hline Ginseng (876) & 7 & 1 & 22 & 265 & 57 & 152 & 110 \\
\hline Guizhi (46) & 0 & 22 & l & 20 & 7 & 26 & 24 \\
\hline Jujube (429) & 1 & 265 & 20 & l & 57 & 28 & 105 \\
\hline Coptis chinensis (67) & 0 & 57 & 7 & 57 & 1 & 7 & 54 \\
\hline Pinellia (196) & 1 & 152 & 26 & 28 & 7 & / & 24 \\
\hline Licorice (115) & 0 & 110 & 24 & 105 & 54 & 24 & 1 \\
\hline
\end{tabular}

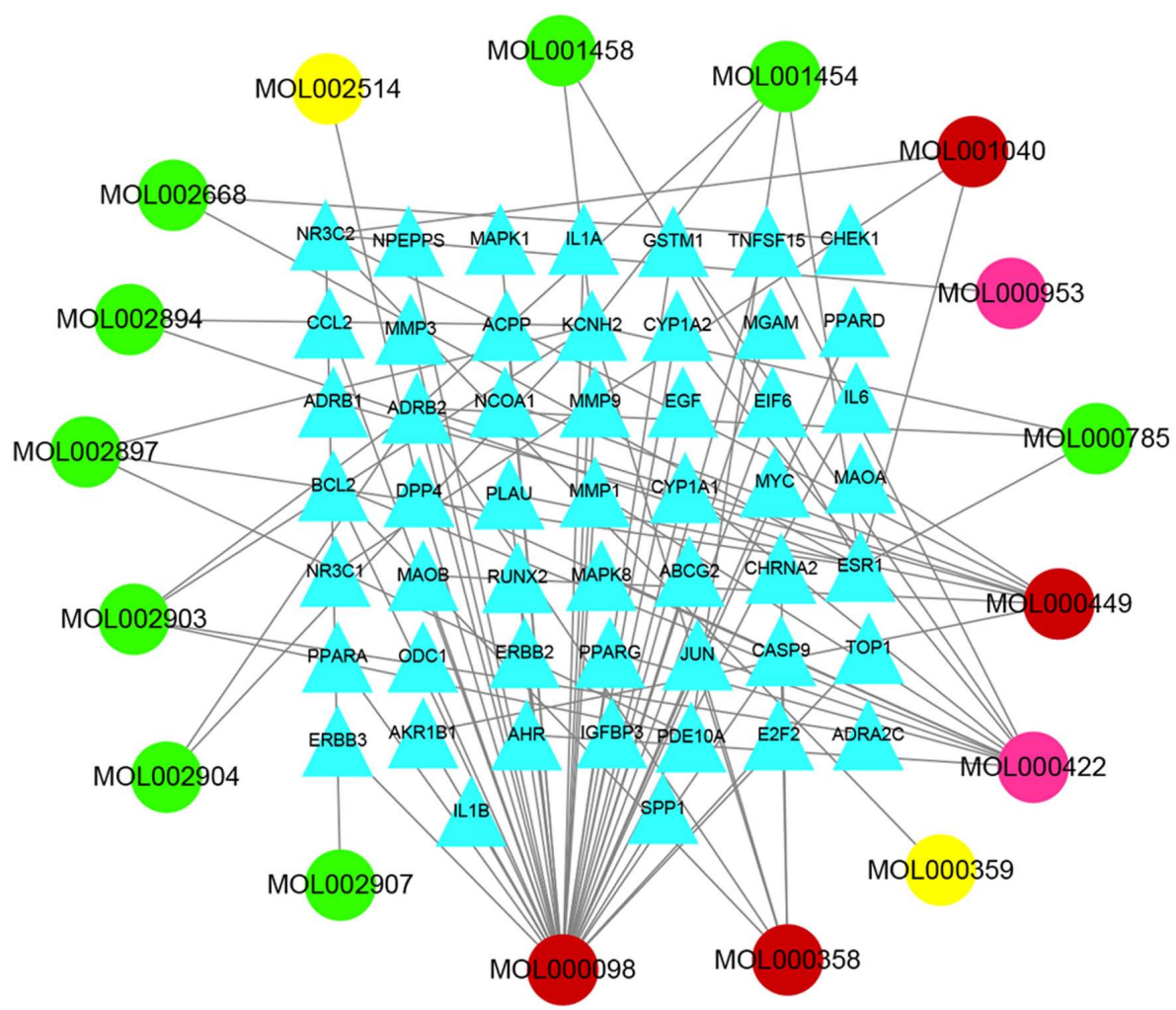

Figure 3. Network of active components of Huang-Lian-Tang and molecular targeting relationships. There are 17 drug nodes (round nodes, dried ginger is yellow, Coptis is green, ume is pink, and various drug collections are red) and 51 molecular nodes (triangular nodes).

patients. The target genes of HLT identified in the present study hold promise for the development of novel targeted therapies for GBM.

Glioma is characterized by rapid growth and resistance to treatment, and patients usually have a poor prognosis (28). Diffuse glioma is the most common intracranial malignancy, accounting for $>60 \%$ of cases (29). GBM is the most common and malignant type of primary brain tumor, accounting for $\sim 80 \%$ of malignant astrocytomas (30). GBM cells vary in shape and size, which is known as glioblastoma polymorphisms (30). GBM may develop rapidly from undiagnosed, less malignant precursor lesions or may progress slowly from preexisting low-grade glioma (31). In spite of the progress in the treatment of GBM, including chemotherapy, the prognosis remains poor $(32,33)$. Therefore, it is necessary to identify potential pathways for the development of this cancer type and to prevent the occurrence of GBM.

In the previous decades, scientists have studied the potential activity of drugs and conducted basic research or clinical trials for tumor treatment (34). The introduction of the concept of big data and the continuous development of pharmacology has provided the opportunity to analyze the relationship between drugs and molecular targets of diseases in silico (35). Network pharmacology is helpful for exploring multi-channel signaling pathway regulation, improve drug efficacy and the success rate of clinical trials and reduce drug development costs. Chinese herbal medicines and plant ingredients have positive prospects in the treatment of a variety of complex diseases $(36,37)$. Network pharmacology has been extensively applied to study the 

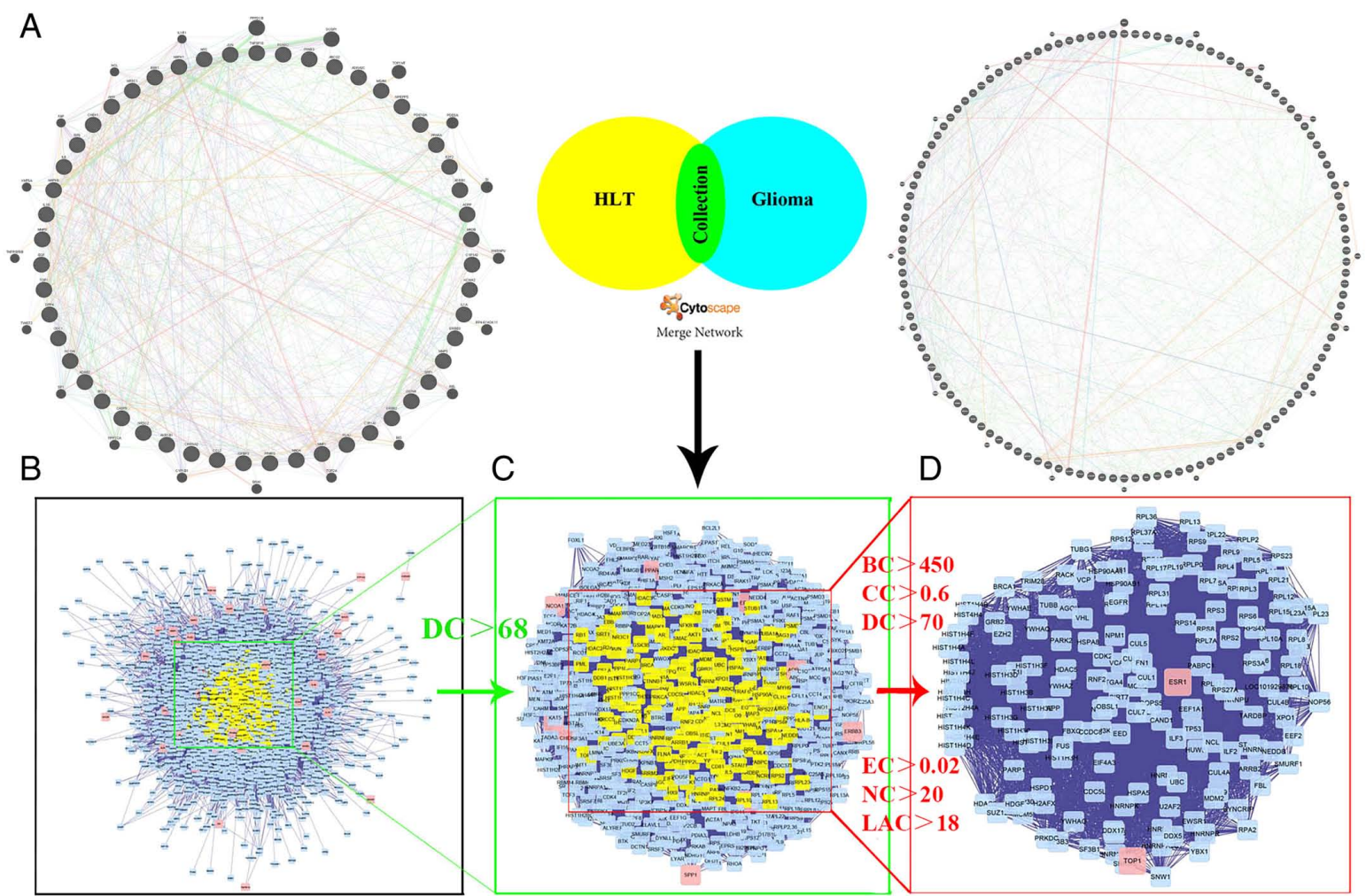

2954 nodes and 72642 edges

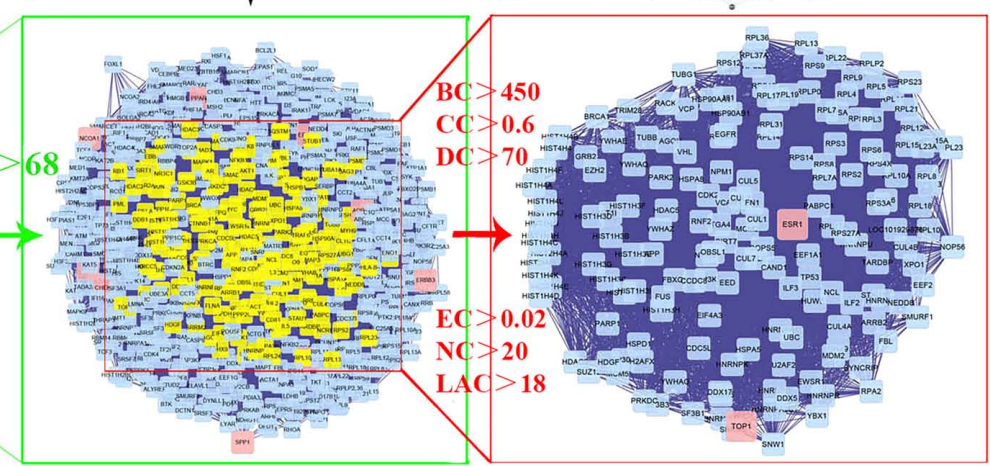

623 nodes and 25934 edges

171 nodes and 6309 edges

Figure 4. Identification of a core PPI network for the mechanism/drug-target interactions. (A) Construction of two PPI networks, one for HLT targets and the other for glioblastoma multiforme. (B) The interactive PPI network of HLT and glioblastoma multiforme targets comprising 2,954 nodes and 72,642 edges. (C) PPI network of significant proteins extracted from B; this network comprises 623 nodes and 25,934 edges. (D) PPI network of significant proteins extracted from C; this network is made up of 171 nodes and 6,309 edges. BC, betweenness centrality; CC, closeness centrality; DC, degree centrality; EC, eigenvector centrality; NC, network centrality; LAC, local average connectivity; PPI, protein-protein interaction; HLT, Huang-Lian-Tang.

biological mechanisms of certain Traditional Chinese Medicine prescriptions and ingredients $(38,39)$. Therefore, the network pharmacology method was used to understand the biological mechanisms of action HLT against GBM at the molecular level.

In southeast Asia, HLT has a significant role in the treatment of brain diseases. Studies have indicated that HLT is able to nourish the nerve cell, restore brain function and improve blood circulation in the brain (40). Studies have also suggested that HLT is an anti-apoptotic, anti-oxidant and anti-inflammatory agent (41-43). HLT has also been indicated to promote neurotrophic factor expression (44) and regulate neurogenesis of neural precursor cells (45), which is conducive to the recovery of neural function. In the present study, not only network pharmacology, but also bioinformatics were used, and a topology analysis was performed. The central network with 171 nodes and 6,309 edges was screened out. GO analysis was performed and HLT was indicated to be involved in numerous biological processes associated with tumorigenesis and development, including RNA polymerase transcription factor complex and response to nutrient levels. The KEGG pathway analysis of the core PPI network was used to identify pathways by which HLT exerts its effects in treating GBM by regulating tumorigenesis and progression. According to the P-value of each enriched pathway and its role in GBM, there were seven related signaling pathways that were significant, and these pathways were all closely associated with the amino acid metabolism of tumorigenesis and progression. It is well known that cancer cells promote the 'Warburg effect' (46), which is enhanced glycolysis or aerobic glycolysis, even when the surrounding oxygen supply is sufficient. In addition, it has been determined that dysfunctional anabolism/catabolism of fatty acids and amino acids, particularly glutamine, serine and glycine, have a role as metabolic regulators in supporting cancer cell growth $(47,48)$. Furthermore, extensive crosstalk was reported between dysfunctional metabolic networks and cancer cell signaling $(49,50)$.

The occurrence and development of tumors are related to a large number of cellular processes, which ultimately lead to the formation or recurrence of tumors, including the induction of cell cycle processes, evasion of apoptotic programs and activation of cell survival pathways (51). In the present study, it was observed that HLT disrupts the process by which these cancer cells are being established, thereby limiting their growth in vitro and in vivo. It remains elusive how HLT specifically reduces tumor growth under normal cellular conditions, so that it does not cause any systemic imbalances or serious side effects for patients. However, it may be speculated that individual active ingredients in the prescription have a mutual synergy, and that the tumor cells are affected by drugs at a concentration below the lethal dose for normal cells.

There are still certain deficiencies in the present study that require to be addressed. First, the present study was only performed in silico. In the next step, a pharmacology model 


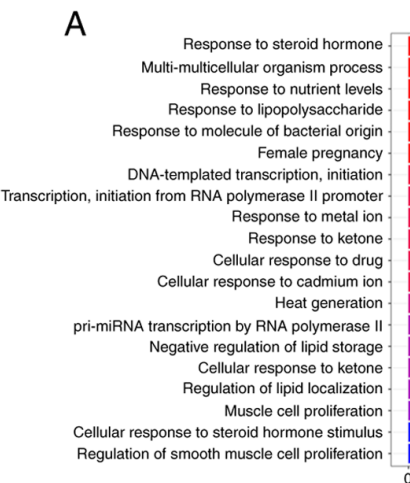

C

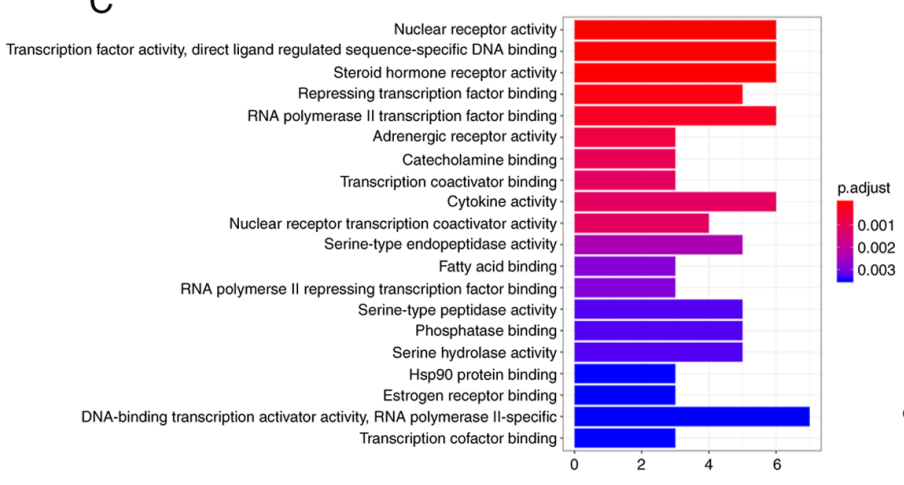

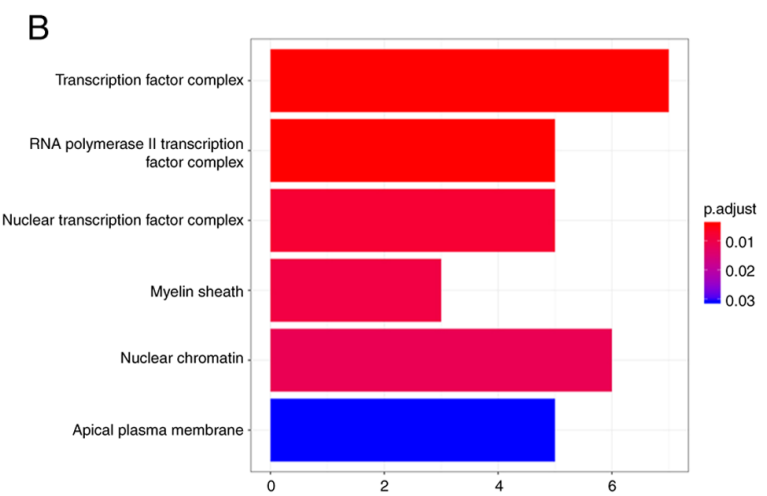

$\mathrm{D}$

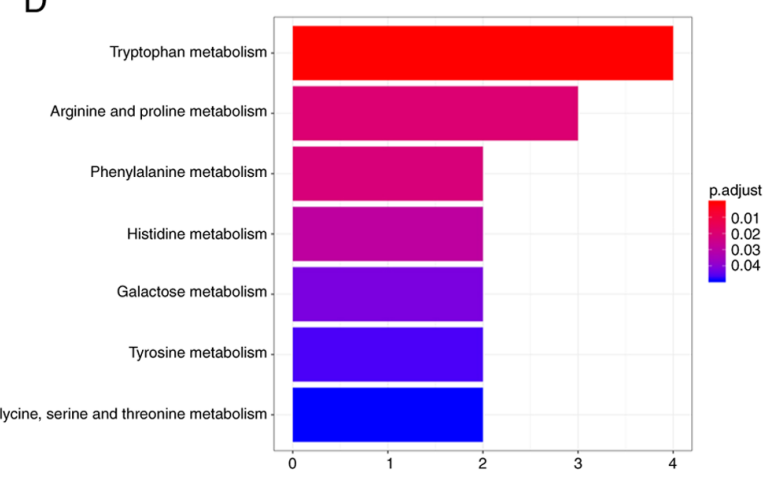

Figure 5. GO analysis and KEGG pathway analyses were performed on screened genes. The top 10 terms in (A-C) the GO categories (A) biological process, (B) cellular component and (C) molecular function, and (D) KEGG pathways. GO, Gene Ontology; KEGG, Kyoto Encyclopedia of Genes and Genomes. P. adjust, adjusted P-value; HSP, heat shock protein.

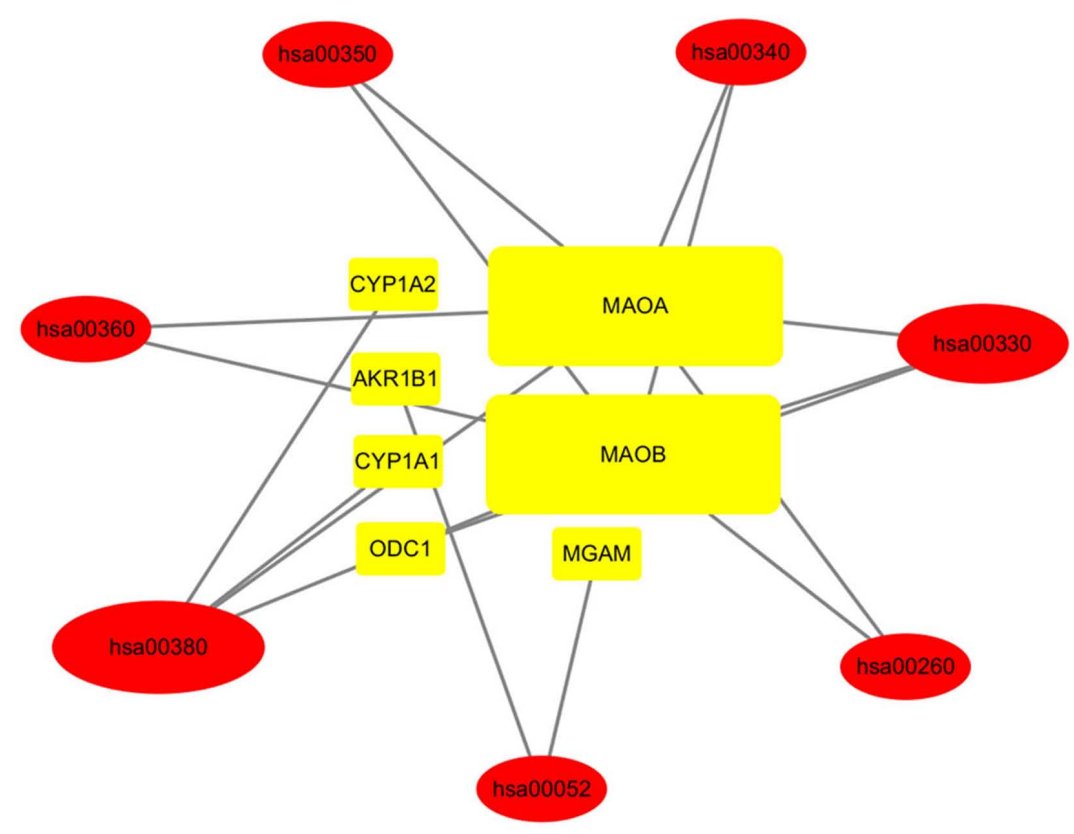

Figure 6. Network of compounds of Huang-Lian-Tang-drug targets-atherosclerosis targets-signaling pathways. A total of 7 genes (oval nodes) and 7 Kyoto Encyclopedia of Genes and Genomes signaling pathways (rectangular nodes) associated with them were obtained. The size of the nodes is proportional to the number of adjacent nodes. Hsa, Homo sapiens. MAOA, Monoamine Oxidase A; MAOB, Monoamine Oxidase B; MGAM, Maltase-Glucoamylase; CYP1A2, Cytochrome P450 Family 1 Subfamily A Member 2; CYP1A1, Cytochrome P450 Family 1 Subfamily A Member 1; AKR1B1, Aldo-Keto Reductase Family 1 Member B; ODC1, Ornithine Decarboxylase 1.

will be built to further confirm the therapeutic effect of HLT on GBM through basic experiments. Furthermore, the optimal dosage of HLT for treating GBM patients and contraindications require to be established. In the future, in-depth research on the most important targets or pathways of the final drug candidates will be performed.

In conclusion, the present network pharmacology analysis on the effect of HLT in GBM indicated that it may be a promising 
chemotherapeutic drug whether it is able to slow/inhibit tumor growth and recurrence and whether it is used as an adjuvant treatment for patients subjected to other standard treatments remains to be elucidated. HLT have few side effects (52), so they may be used in GBM-associated clinical trials.

\section{Acknowledgements}

Not applicable.

\section{Funding}

No funding was received.

\section{Availability of data and materials}

The datasets generated and analyzed during the current study are available in the [GEO and TCM] repositories, [https://www. ncbi.nlm.nih.gov/geo/;https://tcmspw.com/tcmsp.php].

\section{Authors' contributions}

Conceptualization: WRL, WHX and CYL. Data collection and interpretation: WRL, WHX, JFX and RYH. Formal analysis: HNH, XJL, WRL, WHX, JFX and KH. Funding acquisition: HNH, XJL, WRL and WHX. Methodology: WRL, WHX, CYL, JFX and RYH. Project administration: HNH, XJL, KH, HNH, XJL, JFX and RYH. Resources: HNH, XJL, JFX, RYH and KH. Supervision: JFX, KH and RYH. Writing: WRL, WHX, CYL, JFX, RYH and KH. Review and editing: HNH and XJL. Responsible for all aspects of the work to ensure that issues related to the accuracy or completeness of any part of the work are properly investigated and resolved: $\mathrm{HNH}$ and XJL. All authors were involved in writing the manuscript and read and approved the final version of the manuscript.

\section{Ethics approval and consent to participate}

Not applicable.

\section{Patient consent for publication}

Not applicable.

\section{Competing interests}

The authors declare that they have no competing interests.

\section{References}

1. Wen PY and Kesari S: Malignant gliomas in adults. N Engl J Med 359: 492-507, 2008.

2. Ostrom QT, Gittleman H, Farah P, Ondracek A, Chen Y, Wolinsky Y, Stroup NE, Kruchko C and Barnholtz-Sloan JS: CBTRUS statistical report: Primary brain and central nervous system tumors diagnosed in the United States in 2006-2010. Neuro Oncol 15 (Suppl 2): ii1-ii56, 2013.

3. Liu BL, Cheng JX, Zhang $X$ and Zhang W: Controversies concerning the application of brachytherapy in central nervous system tumors. J Cancer Res Clin Oncol 136: 173-185, 2010.

4. Zong G, Wang H, Li J, Xie Y, Bian E and Zhao B: Inhibition of GPR137 expression reduces the proliferation and colony formation of malignant glioma cells. Neurol Sci 35: 1707-1714, 2014.
5. Baskar R, Lee KA, Yeo R and Yeoh KW: Cancer and radiation therapy: Current advances and future directions. Int J Med Sci 9: 193-199, 2012.

6. Rivera Vargas T and Apetoh L: Danger signals: Chemotherapy enhancers? Immunol Rev 280: 175-193, 2017.

7. PDQ Pediatric Treatment Editorial Board: Childhood brain stem glioma treatment (PDQ(R)): Patient Version. In: PDQ Cancer Information Summaries [Internet]. National Cancer Institute (US), Bethesda, MD, 2002.

8. Tao W, Luo X, Cui B, Liang D, Wang C, Duan Y, Li X, Zhou S, Zhao M, Li Y, et al: Practice of traditional Chinese medicine for psycho-behavioral intervention improves quality of life in cancer patients: A systematic review and meta-analysis. Oncotarget 6: 39725-39739, 2015.

9. Chang WH, Chen CH, Gau RJ, Lin CC, Tsai CL, Tsai K and Lu FJ: Effect of baicalein on apoptosis of the human Hep G2 cell line was induced by mitochondrial dysfunction. Planta Med 68: 302-306, 2002.

10. Ma Z, Otsuyama K, Liu S, Abroun S, Ishikawa H, Tsuyama N, Obata M, Li FJ, Zheng X, Maki Y, et al: Baicalein, a component of Scutellaria radix from Huang-lian-jie-du-tang (HLJDT), leads to suppression of proliferation and induction of apoptosis in human myeloma cells. Blood 105: 3312-3318, 2005.

11. Lee WR, Shen SC, Lin HY, Hou WC, Yang LL and Chen YC: Wogonin and fisetin induce apoptosis in human promyeloleukemic cells, accompanied by a decrease of reactive oxygen species, and activation of caspase 3 and $\mathrm{Ca}(2+)$-dependent endonuclease. Biochem Pharmacol 63: 225-236, 2002.

12. Jia KK, Ding H, Yu HW, Dong TJ, Pan Y and Kong LD: Huanglian-wendan decoction inhibits NF- $\mathrm{B} / \mathrm{NLRP} 3$ inflammasome activation in liver and brain of rats exposed to chronic unpredictable mild stress. Mediators Inflamm 2018: 3093516, 2018.

13. Gu XR, Fang SY, Ren W, Wang HJ, Yang J, Si N, Bian BL and Zhao HY: Pharmacodynamics of Huanglian Jiedu decoction in Alzheimer's disease (AD) model rats and effect on improvement of inflammation microenvironment in brain. Zhongguo Zhong Yao Za Zhi 43: 3006-3011, 2018 (In Chinese).

14. Hu S, Chen CW, Chen ST, Tsui KH, Tang TK, Cheng HT, Hwang GS, Yu JW, Li YC, Wang PS and Wang SW: Inhibitory effect of berberine on interleukin-2 secretion from PHA-treated lymphocytic Jurkat cells. Int Immunopharmacol 66: 267-273, 2019.

15. Kang JX, Liu J, Wang J, He C and Li FP: The extract of Huanglian, a medicinal herb, induces cell growth arrest and apoptosis by upregulation of interferon-beta and TNF-alpha in human breast cancer cells. Carcinogenesis 26: 1934-1939, 2005.

16. Gong B, Kao Y, Zhang C, Zhao H, Sun F and Gong Z: Exploring the pharmacological mechanism of the herb pair 'HuangLian-GanJiang' against colorectal cancer based on network pharmacology. Evid Based Complement Alternat Med 2019: 2735050, 2019.

17. Dai Y, Sun L and Qiang W: A new strategy to uncover the anticancer mechanism of Chinese compound formula by integrating systems pharmacology and bioinformatics. Evid Based Complement Alternat Med 2018: 6707850, 2018.

18. Ru J, Li P, Wang J, Zhou W, Li B, Huang C, Li P, Guo Z, Tao W, Yang Y, et al: TCMSP: A database of systems pharmacology for drug discovery from herbal medicines. J Cheminform 6: 13, 2014.

19. Hsu YL, Kuo PL, Tzeng TF, Sung SC, Yen MH, Lin LT and Lin CC: Huang-lian-jie-du-tang, a traditional Chinese medicine prescription, induces cell-cycle arrest and apoptosis in human liver cancer cells in vitro and in vivo. J Gastroenterol Hepatol 23: e290-e299, 2008.

20. Wang N, Feng Y, Tan HY, Cheung F, Hong M, Lao L and Nagamatsu T: Inhibition of eukaryotic elongation factor-2 confers to tumor suppression by a herbal formulation Huanglian-Jiedu decoction in human hepatocellular carcinoma. J Ethnopharmacol 164: 309-318, 2015.

21. Fang H, Wang Y, Yang T, Ga Y, Zhang Y, Liu R, Zhang W and Zhao J: Bioinformatics analysis for the antirheumatic effects of Huang-lian-jie-du-tang from a network perspective. Evid Based Complement Alternat Med 2013: 245357, 2013.

22. Xu T, Ma C, Fan S, Deng N, Lian Y, Tan L, Du W, Zhang S, Liu S, Ren B, et al: Systematic understanding of the mechanism of baicalin against ischemic stroke through a network pharmacology approach. Evid Based Complement Alternat Med 2018: 2582843, 2018.

23. Martin A, Ochagavia ME, Rabasa LC, Miranda J, Fernandez-deCossio J and Bringas R: BisoGenet: A new tool for gene network building, visualization and analysis. BMC Bioinformatics 11: 91, 2010. 
24. Tang Y, Li M, Wang J, Pan Y and Wu FX: CytoNCA: A cytoscape plugin for centrality analysis and evaluation of protein interaction networks. Biosystems 127: 67-72, 2015.

25. Barabasi AL, Gulbahce N and Loscalzo J: Network medicine: A network-based approach to human disease. Nat Rev Genet 12 56-68, 2011.

26. Bader GD and Hogue CW: An automated method for finding molecular complexes in large protein interaction networks. BMC bioinformatics 4: 2, 2003

27. Huang da W, Sherman BT and Lempicki RA: Systematic and integrative analysis of large gene lists using DAVID bioinformatics resources. Nat Protoc 4: 44-57, 2009.

28. GLASS Consortium: Glioma through the looking GLASS: Molecular evolution of diffuse gliomas and the glioma longitudinal analysis consortium. Neuro Oncol 20: 873-884, 2018.

29. Louis DN, Perry A, Reifenberger G, von Deimling A, Figarella-Branger D, Cavenee WK, Ohgaki H, Wiestler OD, Kleihues P and Ellison DW: The 2016 world health organization classification of tumors of the central nervous system: A summary. Acta Neuropathol 131: 803-820, 2016.

30. Szopa W, Burley TA, Kramer-Marek G and Kaspera W: Diagnostic and therapeutic biomarkers in glioblastoma: Current status and future perspectives. Biomed Res Int 2017: 8013575, 2017.

31. Sugimoto K, Ideguchi M, Kimura T, Kajiwara K, Imoto $H$, Sadahiro H, Ishii A, Kawano H, Ikeda E and Suzuki M: Epithelioid/rhabdoid glioblastoma: A highly aggressive subtype of glioblastoma. Brain Tumor Pathol 33: 137-146, 2016.

32. Farias-Eisner G, Bank AM, Hwang BY, Appelboom G, Piazza MA, Bruce SS and Sander Connolly E: Glioblastoma biomarkers from bench to bedside: Advances and challenges. $\mathrm{Br}$ J Neurosurg 26: 189-194, 2012

33. Li Y, Ren Z, Peng Y, Li K, Wang X, Huang G, Qi S and Liu Y: Classification of glioma based on prognostic alternative splicing. BMC Med Genomics 12: 165, 2019.

34. Terstappen GC, Schlupen C, Raggiaschi R and Gaviraghi G: Target deconvolution strategies in drug discovery. Nat Rev Drug Discov 6: 891-903, 2007.

35. Zhang GB, Li QY, Chen QL and Su SB: Network pharmacology: A new approach for chinese herbal medicine research. Evid Based Complement Alternat Med 2013: 621423, 2013.

36. Sang Z, Wang K, Shi J, Liu W, Cheng X, Zhu G, Wang Y, Zhao Y, Qiao Z, Wu A and Tan Z: The development of advanced structural framework as multi-target-directed ligands for the treatment of Alzheimer's disease. Eur J Med Chem 192: 112180, 2020

37. Wang X, Wu W, Zhang J, Gao L, Zhang L, Long H, Hou J, Wu W and Guo D: An integrated strategy for holistic quality identification of Chinese patent medicine: Liuwei dihuang pills as a case study. Phytochem Anal: Mar 4, 2020 (Epub ahead of print) doi: $10.1002 /$ pca. 2927.

38. Zhu N and Hou J: Exploring the mechanism of action xianlingubao prescription in the treatment of osteoporosis by network pharmacology. Comput Biol Chem 85: 107240, 2020.
39. Zhou X, Hong Y and Zhan Y: Karacoline, identified by network pharmacology, reduces degradation of the extracellular matrix in intervertebral disc degeneration via the NF- $\mathrm{\kappa B}$ signaling pathway. J Pharm Anal 10: 13-22, 2020.

40. Gu Y, Chen J and Shen J: Herbal medicines for ischemic stroke: Combating inflammation as therapeutic targets. J Neuroimmune Pharmacol 9: 313-339, 2014.

41. Chen SJ and Cui MC: Systematic understanding of the mechanism of salvianolic acid a via computational target fishing. Molecules 22: 644, 2017.

42. Wang S, Wang H and Lu Y: Tianfoshen oral liquid: A CFDA approved clinical traditional Chinese medicine, normalizes major cellular pathways disordered during colorectal carcinogenesis. Oncotarget 8: 14549-14569, 2017.

43. Wang X, Yu S, Jia Q, Chen L, Zhong J, Pan Y, Shen P, Shen Y, Wang S, Wei Z, et al: NiaoDuQing granules relieve chronic kidney disease symptoms by decreasing renal fibrosis and anemia. Oncotarget 8: 55920-55937, 2017.

44. Xu M, Chen X, Gu Y, Peng T, Yang D, Chang RC, So KF, Liu K and Shen J: Baicalin can scavenge peroxynitrite and ameliorate endogenous peroxynitrite-mediated neurotoxicity in cerebral ischemia-reperfusion injury. J Ethnopharmacol 150: 116-124, 2013.

45. Cao Y, Mao X, Sun C, Zheng P, Gao J, Wang X, Min D, Sun H, Xie N and Cai J: Baicalin attenuates global cerebral ischemia/reperfusion injury in gerbils via anti-oxidative and anti-apoptotic pathways. Brain Res Bull 85: 396-402, 2011.

46. Schwartz L, Supuran CT and Alfarouk KO: The warburg effect and the hallmarks of cancer. Anticancer Agents Med Chem 17: 164-170, 2017.

47. Colomer C, Margalef P, Villanueva A, Vert A, Pecharroman I, Solé L, González-Farré M, Alonso J, Montagut C, Martinez-Iniesta $\mathrm{M}$, et al: IKK $\alpha$ kinase regulates the DNA damage response and drives chemo-resistance in cancer. Mol Cell 75: 669-682.e5, 2019

48. Huang Y, Huang Y, Zhang L, Chang A, Zhao P, Chai X and Wang J: Identification of crucial genes and prediction of small molecules for multidrug resistance of Hodgkin's lymphomas. Cancer Biomark 23: 495-503, 2018.

49. Hensley CT, Wasti AT and DeBerardinis RJ: Glutamine and cancer: Cell biology, physiology, and clinical opportunities. J Clin Invest 123: 3678-3684, 2013.

50. SrinivasanS, Guha M,Kashina A and Avadhani NG: Mitochondrial dysfunction and mitochondrial dynamics-The cancer connection. Biochim Biophys Acta Bioenerg 1858: 602-614, 2017.

51. Korf BR: Neurofibromatosis. Handb Clin Neurol 111: 333-340, 2013.

52. Singh A and Zhao K: Treatment of insomnia with traditional Chinese herbal medicine. Int Rev Neurobiol 135: 97-115, 2017.

This work is licensed under a Creative Commons Attribution-NonCommercial-NoDerivatives 4.0 International (CC BY-NC-ND 4.0) License. 